Praxishandbuch Fernsehen 
Eric Karstens • Jörg Schütte

\section{Praxishandbuch Fernsehen}

Wie TV-Sender arbeiten

3., aktualisierte Auflage

Springer VS 


\section{Eric Karstens}

Jörg Schütte

Köln, Deutschland

Die Deutsche Nationalbibliothek verzeichnet diese Publikation in der Deutschen Nationalbibliografie; detaillierte bibliografische Daten sind im Internet über http://dnb.d-nb.de abrufbar.

\section{Springer VS}

(c) Springer Fachmedien Wiesbaden 2005, 2010, 2013

Das Werk einschließlich aller seiner Teile ist urheberrechtlich geschützt. Jede Verwertung, die nicht ausdrücklich vom Urheberrechtsgesetz zugelassen ist, bedarf der vorherigen Zustimmung des Verlags. Das gilt insbesondere für Vervielfältigungen, Bearbeitungen, Übersetzungen, Mikroverfilmungen und die Einspeicherung und Verarbeitung in elektronischen Systemen.

Die Wiedergabe von Gebrauchsnamen, Handelsnamen, Warenbezeichnungen usw. in diesem Werk berechtigt auch ohne besondere Kennzeichnung nicht zu der Annahme, dass solche Namen im Sinne der Warenzeichen- und Markenschutz-Gesetzgebung als frei zu betrachten wären und daher von jedermann benutzt werden dürften.

Gedruckt auf säurefreiem und chlorfrei gebleichtem Papier

Springer VS ist eine Marke von Springer DE. Springer DE ist Teil der Fachverlagsgruppe Springer Science+Business Media.

www.springer-vs.de 


\section{Inhalt}

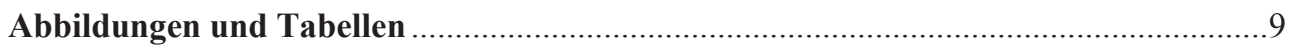

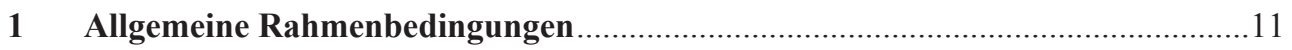

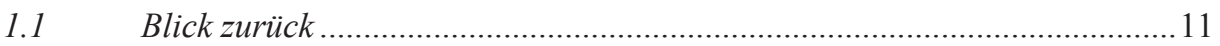

1.1.1 Die Anfänge in den vierziger und fünfziger Jahren................................... 12

1.1.2 „Adenauer-Fernsehen“, ZDF und die Dritten Programme ........................... 14

1.1.3 Programm und Politik: Die sechziger und siebziger Jahre .......................... 17

1.1.4 Das Privatfernsehen: Fernsehen als Markt ................................................ 19

1.1.5 Krise und Konzentration: Die Fernsehlandschaft ab 2000 ..........................22

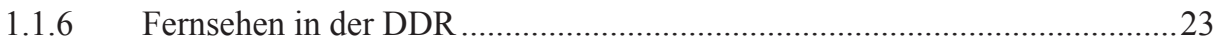

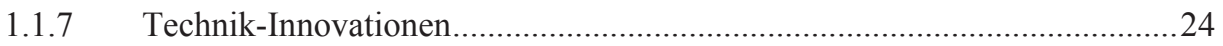

1.2 Rechtliche und politische Grundlagen......................................................26

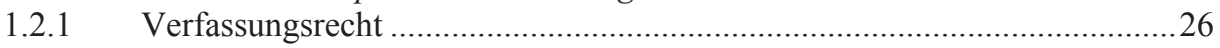

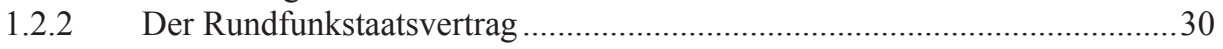

1.2.3 Die Landesmediengesetze und die Landesmedienanstalten ..........................37

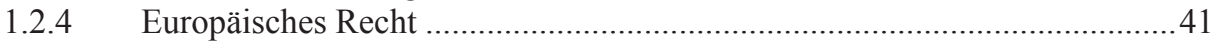

1.2.5 Kontrolle und Finanzierung des öffentlich-rechtliche Rundfunks.................42

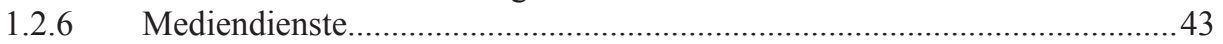

1.3 Rechtliche Vorschriften und Rahmenbedingungen .....................................4

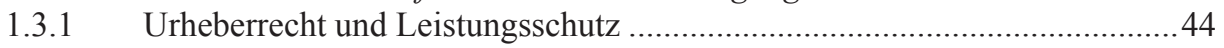

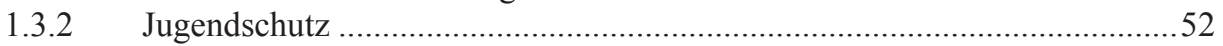

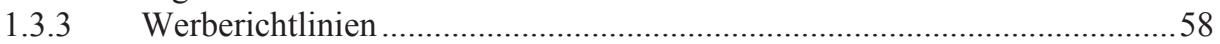

1.3.4 Spezielle Vorschriften für den journalistisch-redaktionellen Bereich ..........64

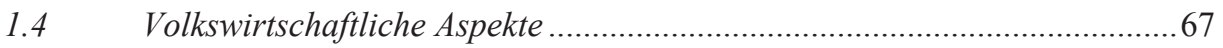

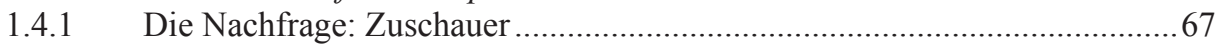

1.4.2 Die Nachfrage: Werbekunden .............................................................. 72

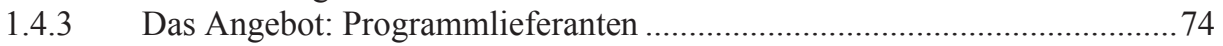

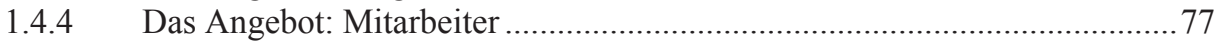

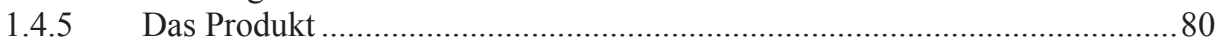

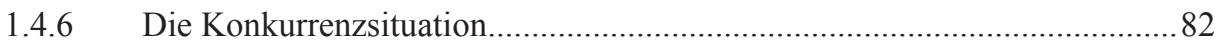

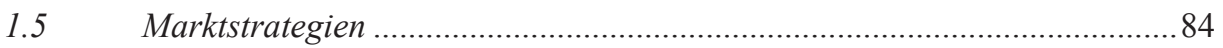

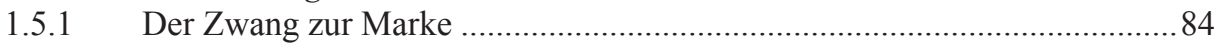

1.5.2 Auf dem Weg zur Marke: Strategien im Zuschauermarkt............................86

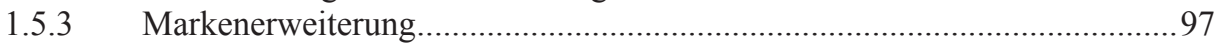

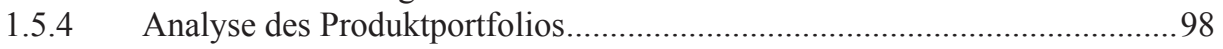

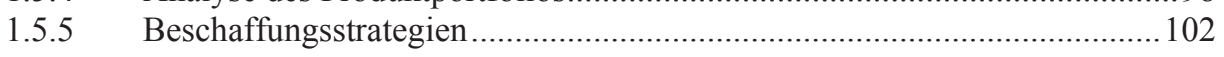




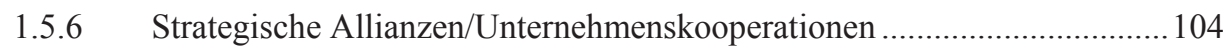

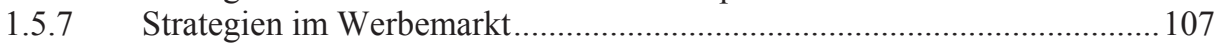

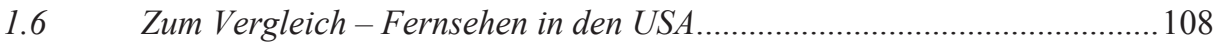

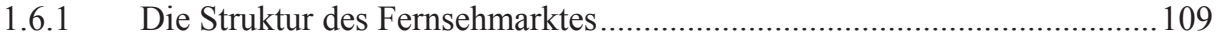

1.6.2 Das Kabelfernsehen............................................................................. 111

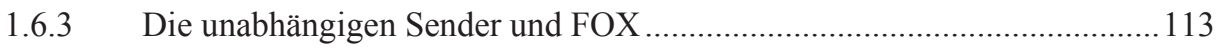

1.6.4 Das öffentliche Fernsehen .................................................................. 114

1.6.5 Programm-Markt und Programm-Produktion ........................................ 115

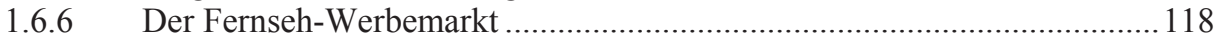

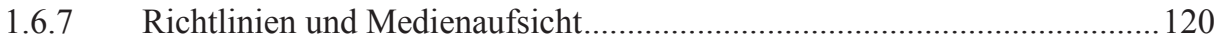

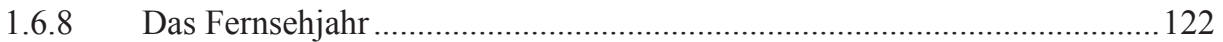

1.6.9 Zuschauerforschung und Einschaltquoten ............................................ 124

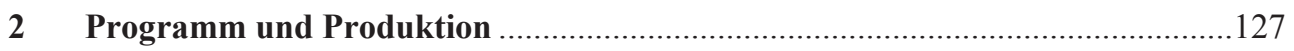

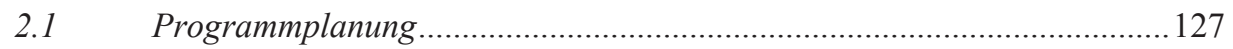

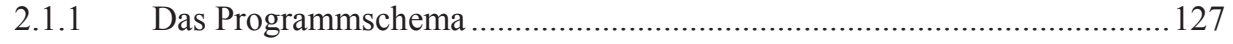

2.1.2 Programmschema-Philosphien und -Ziele............................................. 130

2.1.3 Instrumente der Schemaplanung............................................................ 132

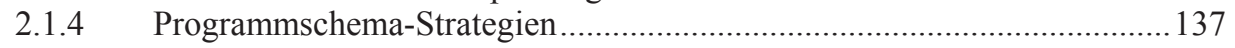

2.1.5 Programmplanung in der Praxis ................................................................. 142

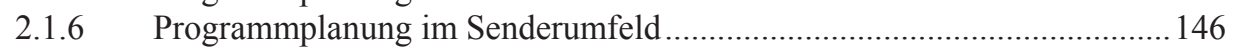

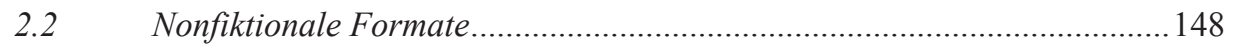

2.2.1 Die Funktionsweise nonfiktionaler Programme ......................................... 149

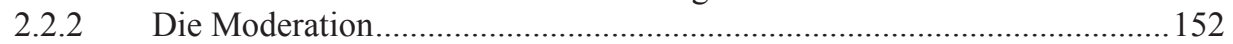

2.2.3 Die Mitarbeiter des Moderators.................................................................. 162

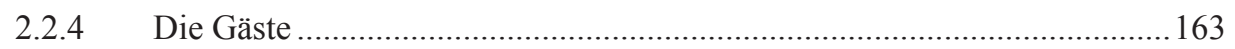

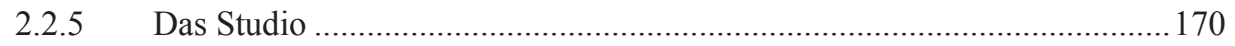

2.2.6 Der Bildbeitrag ....................................................................................... 173

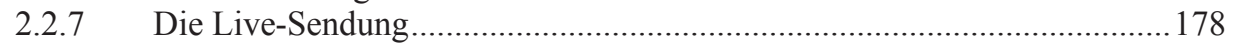

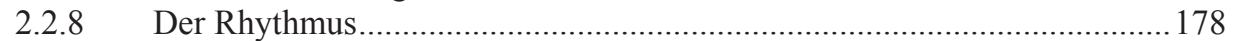

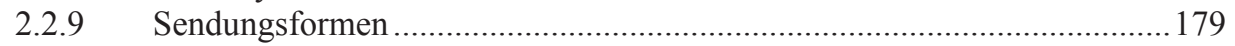

2.3 Von der Idee zur Sendung: Entwicklung und Produktion von

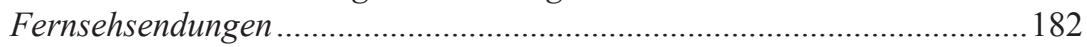

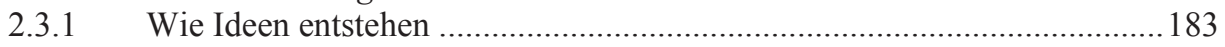

2.3.2 Woher bekommt ein Sender seine Ideen?................................................ 185

2.3.3 Wie wählt ein Sender die Programmideen aus? ........................................... 187

2.3.4 Von der Ideenauswahl zur Umsetzungsentscheidung................................ 189

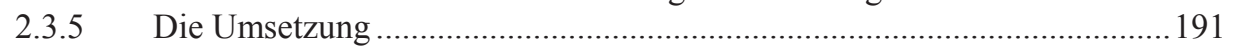

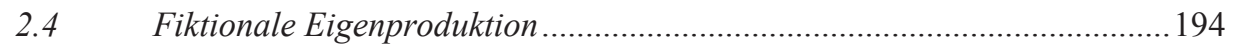

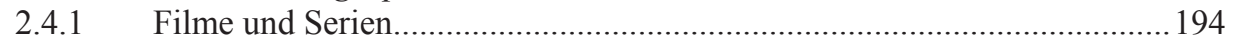

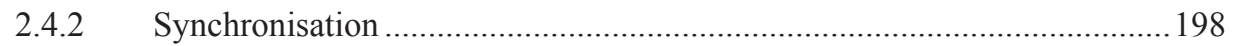

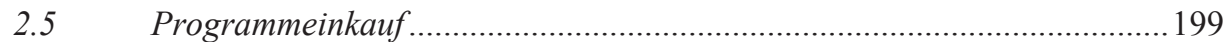

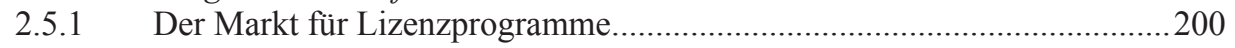

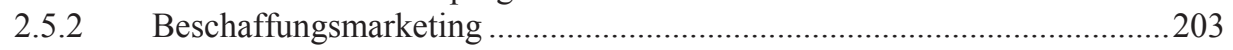


2.5.3 Gattungen von Lizenzprogrammen .......................................................2. 206

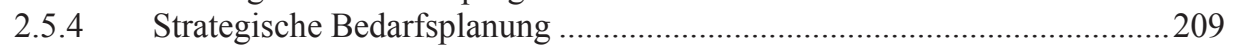

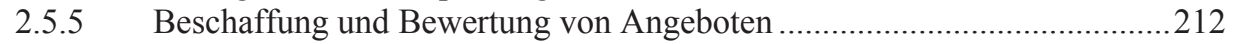

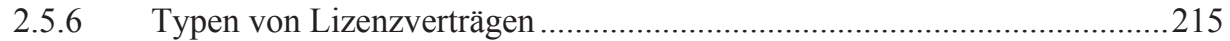

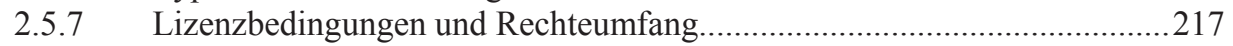

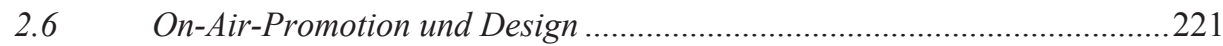

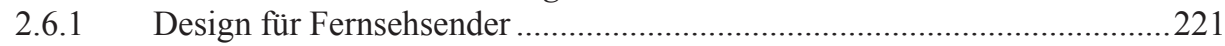

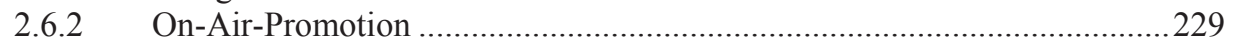

2.6.3 Promotionplanung ...............................................................................22 239

$2.7 \quad$ Exkurs: Management kreativer Prozesse ...................................................2. 241

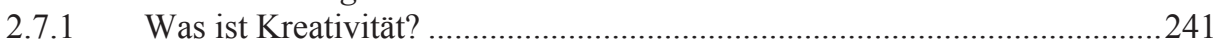

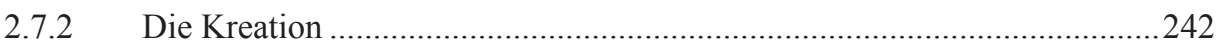

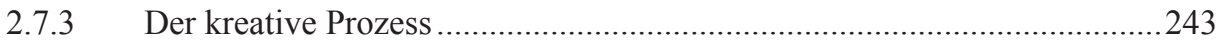

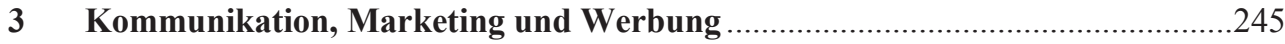

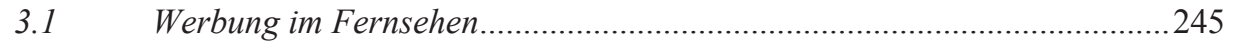

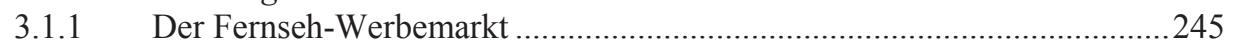

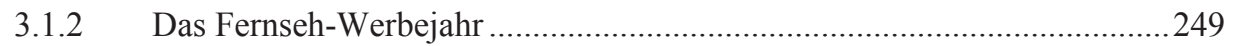

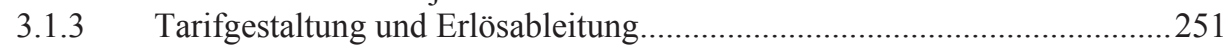

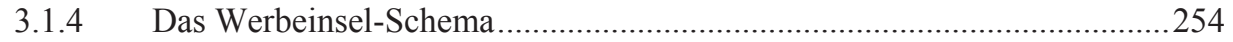

3.1.5 Vermarktung von Fernsehsendern ............................................................2. 258

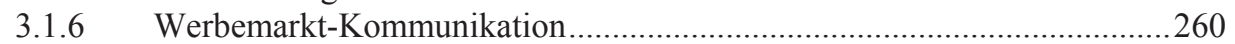

3.1.7 Werbezeitenverkauf und Instrumente des Verkaufs ..................................2.261

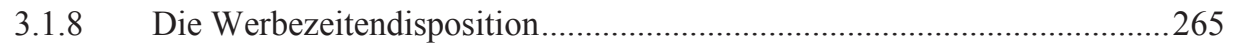

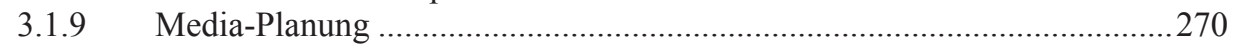

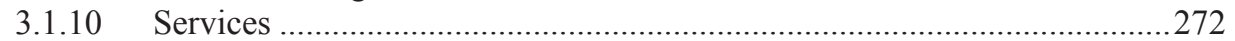

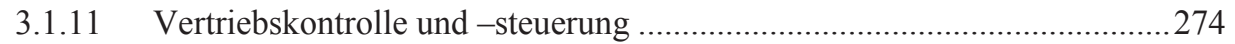

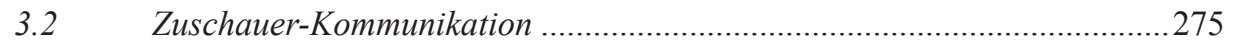

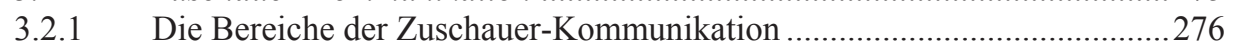

3.2.2 Die Medien der Zuschauerkommunikation ............................................ 279

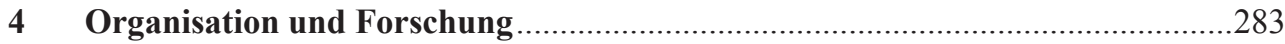

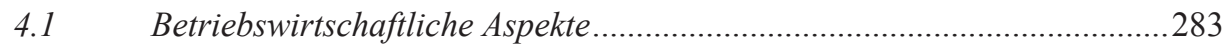

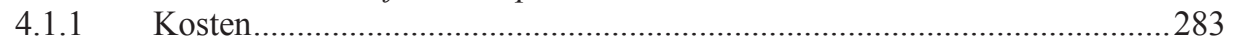

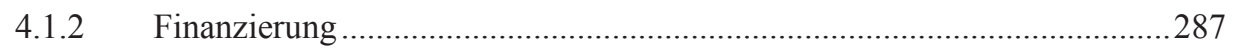

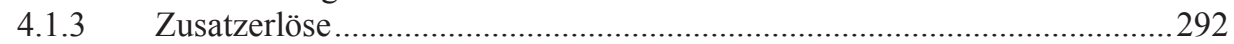

4.1.4 Wirtschaftliche Gesamtbetrachtung.......................................................29

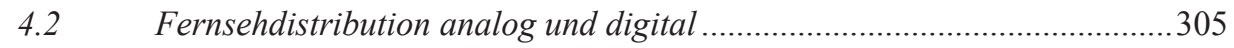

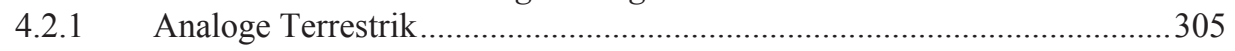

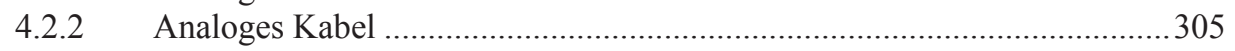

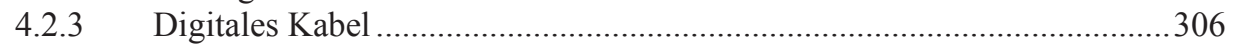

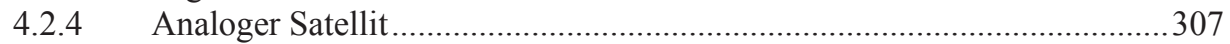

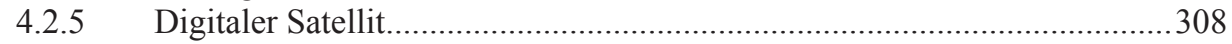




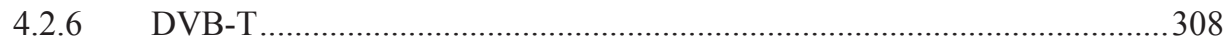

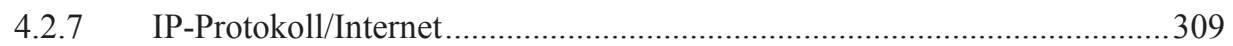

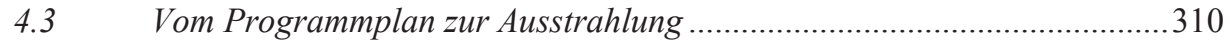

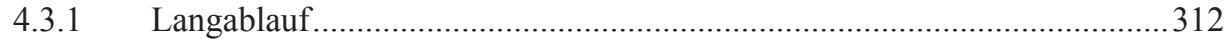

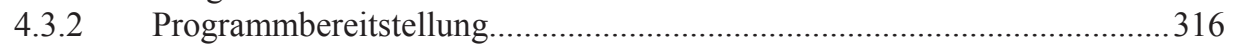

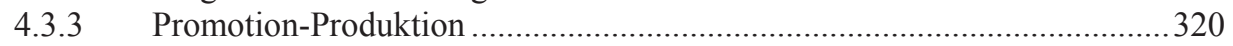

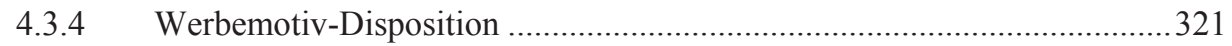

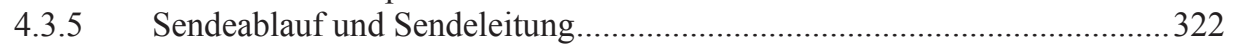

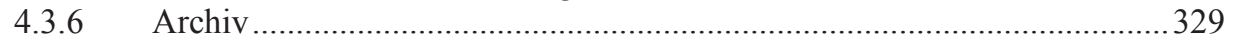

4.4 Fernsehzuschauer- und Marktforschung ....................................................330

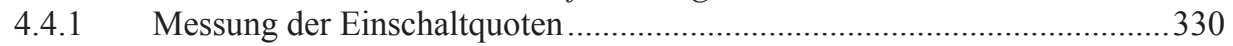

4.4.2 Analyse von Daten der Fernsehforschung ...............................................339

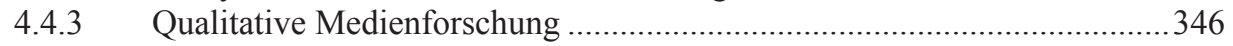

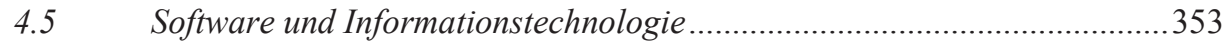

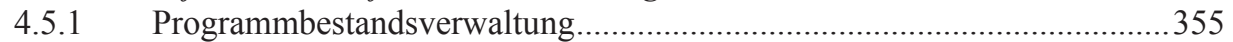

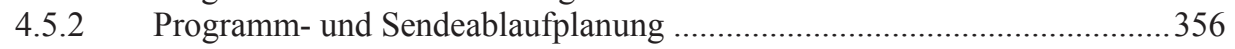

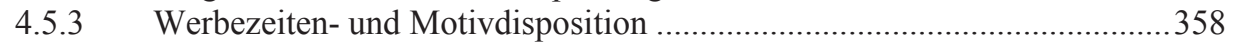

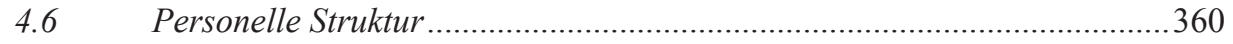

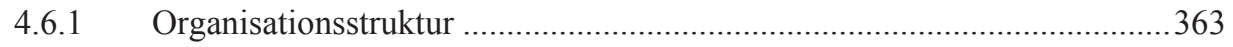

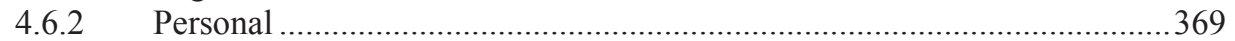

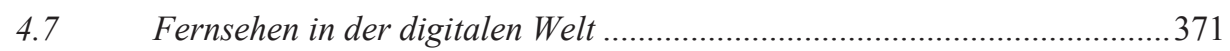

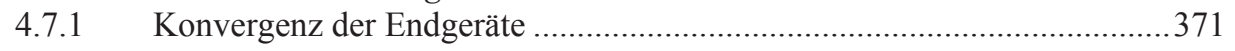

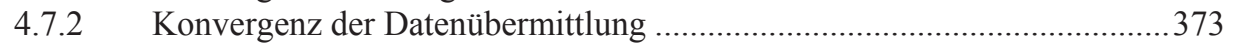

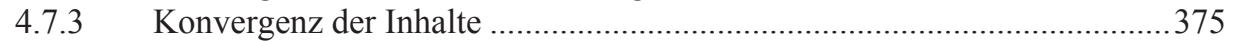

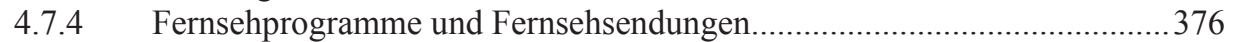

4.7.5 Auswirkungen auf das Programmangebot..................................................... 377

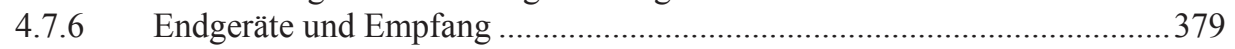

4.7.7 Marktveränderungen durch digitale Fernsehtechniken..............................381

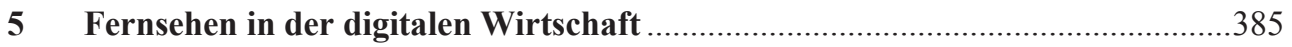

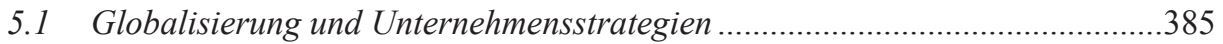

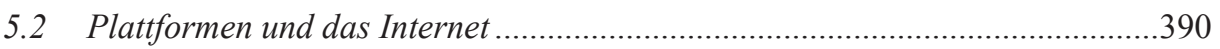

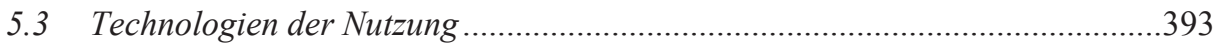

5.4 Governance und politisch-rechtliche Debatten ................................................396

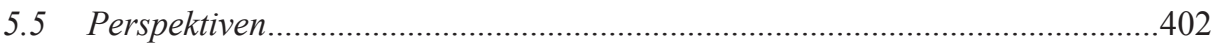

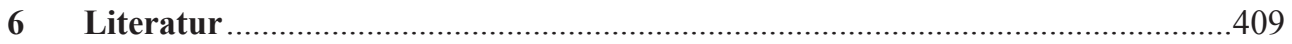

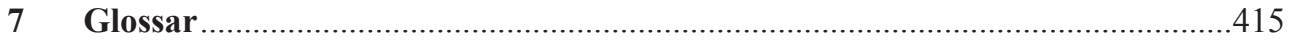

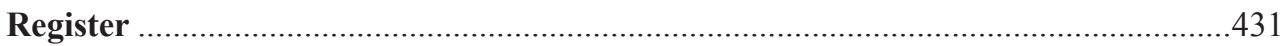




\section{Abbildungen und Tabellen}

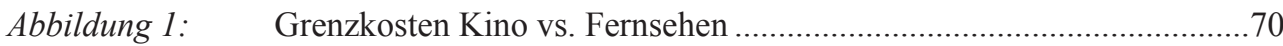

Abbildung 2: $\quad$ Strategische Positionierung ARD / VIVA ............................................ 88

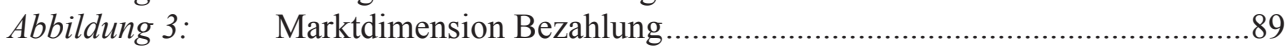

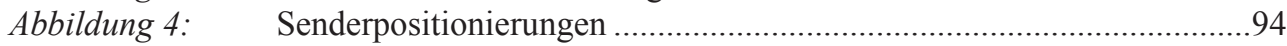

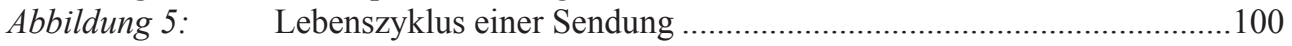

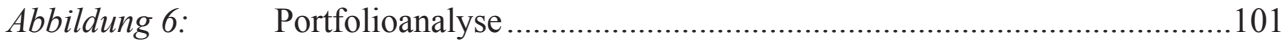

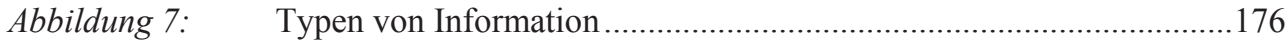

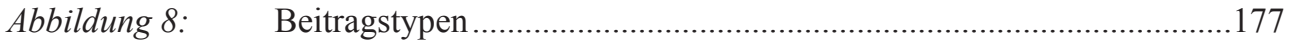

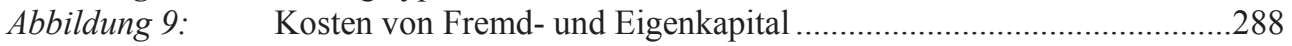

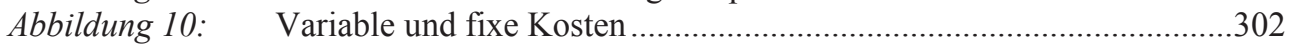

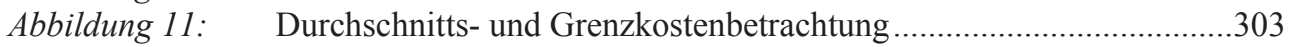

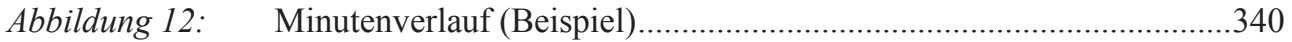

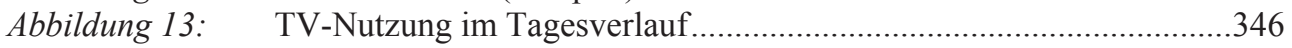

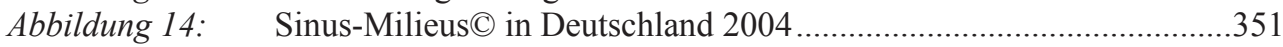

Abbildung 15: $\quad$ Organisationsstruktur eines Fernsehsenders (Beispiel) .........................368

Tabelle 1: $\quad$ Marktanteile der Sender 2008 in Prozent, Zuschauer ab 3 Jahre...................34

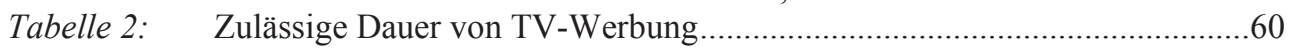

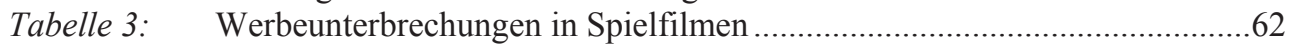

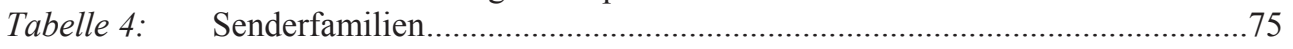

Tabelle 5: $\quad$ Preise für Fernsehlizenzen (Erstverwertung) im internationalen

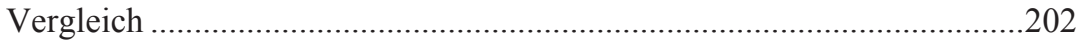

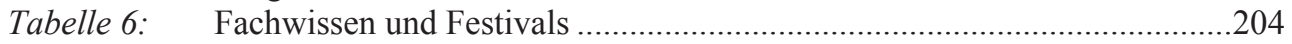

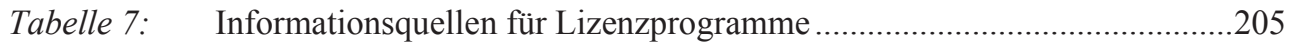

Tabelle 8: $\quad$ Werbe- und Zuschauermarktanteile 2004 ................................................2. 247

Tabelle 9: $\quad$ Die größten Media-Agenturen nach TV-Umsätzen 2002 ..........................248

Tabelle 10: $\quad$ Top 10 der Fernsehwerbung nach Produktgruppen 2002 ............................248

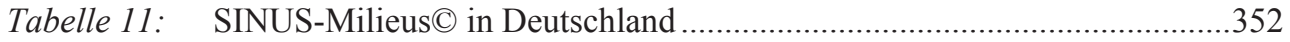

